\title{
STATISTICALLY SIGNIFICANT ESTIMATES OF INFLUENCE OF SOLAR ACTIVITY ON PLANETARY WAVES IN THE MIDDLE ATMOSPHERE OF THE NORTHERN HEMISPHERE AS DERIVED FROM MUAM MODEL DATA
}

\author{
A.V. Koval \\ St. Petersburg State University, \\ Saint Petersburg, Russia, a.v.koval@spbu.ru
}

\begin{abstract}
Numerical simulation has been used to examine the effect of changes in solar activity (SA) in the thermosphere on amplitudes of long-period planetary waves (PW) for the winter period in the Northern Hemisphere. The model of the middle and upper atmosphere (MUAM) is used. It allows simulations of general atmospheric circulation at altitudes $0-300 \mathrm{~km}$. In order to reproduce SA changes, different values of the solar radio flux at a wavelength of $10.7 \mathrm{~cm}$ at an altitude of more than $100 \mathrm{~km}$ are set in the MUAM radiation block. To take into account the effect of charged particles in the ionosphere on the neutral gas dynamics, ionospheric conductivities for different SA levels are included in MUAM. To improve the statistical reliability of the results, two ensembles of model simulations consisting of 16 runs corresponding
\end{abstract}

to the minimum and maximum SA have been obtained. The statistical confidence of average differences in PW amplitudes between high and low SA has been calculated. The results are shown to be reliable in almost the entire altitude range $0-300 \mathrm{~km}$. Results of the simulations have shown for the first time that statistically significant differences in amplitudes of long-period PWs can reach $10-15 \%$ in the middle atmosphere of the Northern Hemisphere, depending on the zonal wave number. At the same time, reflection of PWs at altitudes of lower thermosphere has a significant effect on the PW structure in the middle atmosphere.

Keywords: general circulation, planetary waves, numerical simulation, solar activity.

$\mathrm{km}$ has demonstrated an important role of PWs connecting the upper atmosphere with underlying layers [Krivolutsky et al., 2015].

We employ a numerical model of the middle and upper atmosphere (MUAM) [Pogoreltsev et al., 2007] capable of calculating the general atmospheric circulation at altitudes from the ground to $300-400 \mathrm{~km}$. In recent years, MUAM has yielded significant results, in particular it has shown that SA greatly affects propagation and reflection of both westward PWs [Koval et al., 2018a] and stationary planetary waves (SPW) [Koval et al., 2018b]. This paper examines the influence of changes in the thermosphere caused by SA changes on amplitudes of geopotential height variations generated by long-period PWs (with periods of more than 15 days) in the middle atmosphere of the Northern Hemisphere. MUAM took into account the SA changes only at altitudes above $100 \mathrm{~km}$. At lower altitudes, all calculations were based on identical conditions corresponding to the mean level of SA. This approach was applied to the study of the thermosphere effect alone, without regard to the direct effects of solar radiation on chemical composition, thermal and dynamical characteristics of the middle atmosphere.

\section{METHODS AND APPROACHES MUAM}

MUAM is a modification of the Cologne Model of the Middle Atmosphere developed at the Leipzig Institute for Meteorology COMMA-LIM [Fröhlich et al., 2003]. The main characteristics and physical processes consid- 
ered by MUAM have been described in [Gavrilov et al., 2005; Koval et al., 2018a]. In particular, MUAM involves parameterizing dynamical and thermal effects of internal gravity waves [Gavrilov, 1997] and orographic waves [Gavrilov, Koval, 2013]. At the lower boundary, SPW amplitudes were set based on the geopotential heights in the lower atmosphere for January averaged over 2005-2014 from the Japanese 55-year Reanalysis (JRA-55) [Kobayashi et al., 2015]. The horizontal grid of MUAM has 36 nodes in latitude and 64 nodes in longitude. The vertical grid has 56 levels corresponding to altitudes to $300 \mathrm{~km}$. In addition to the standard radiation scheme used in the original model COMMA-LIM [Fröhlich et al., 2003], MUAM involves parameterizing the thermosphere heating due to EUV. Solar fluxes and absorption coefficients for each spectral interval were calculated by the model [Richards et al., 1994].

The main stages of MUAM initialization are described in [Pogoreltsev et al., 2007; Koval et al., 2018a]. During the first 120 days, the model uses only daily average heating rates. Then it employs daily variations in solar heating and an additional prognostic equation for geopotential at the lower boundary. This prognostic equation should meet the lower boundary condition for waves generated by internal sources. Starting from the 300th model day, MUAM takes into account seasonal variations in solar heating, and the next 90 days are considered as peculiar to conditions in December-February. A change in the initial day of the daily variations in solar heating in MUAM leads to a change in phase of stratospheric vacillations of the zonal-mean zonal wind and PW characteristics [Pogoreltsev, 2007]. Two ensembles of 16 runs of MUAM each (for high and low SA levels respectively) were derived from the change in the initial day of daily variations in solar heating and prognostic equation for geopotential between the 120th and 135 th days with a step of one model day [Koval et al., 2018a, b]. Background conditions for all the model runs were identical. Besides increasing the statistical significance of the results, the averaging over 16 model runs allows us to smooth out such extreme dynamical effects as sudden stratospheric warming events, obtained from individual runs.

\section{Consideration of SA in the model}

MUAM involves parameterizing the thermosphere heating in EUV. The solar radio emission flux with a wavelength of $10.7 \mathrm{~cm}(F 10.7)$ is utilized as an indicator of SA in the MUAM radiation block. $F 10.7$ features the cyclicity with a period of the main 11-year solar cycle [Tapping, 1987; Bruevich, Yakunina, 2015]. Changes in $F 10.7$ correlate well with changes in Wolf numbers [Vitinsky et al., 1986]. From observations made during the last six solar cycles [http://sidc.be/silso/ datafiles] to characterize low, medium, and high levels of SA, $F 10.7$ values equal to 70,130 , and $220 \mathrm{sfu}$ ( 1 $\left.\mathrm{sfu}=10^{-22} \mathrm{~W} /\left(\mathrm{m}^{2} \mathrm{~Hz}\right)\right)$ respectively have been selected. To study the thermospheric effects of SA, different $F 10.7$ values were specified in MUAM only above 100 $\mathrm{km}$. Below $100 \mathrm{~km}$, the calculations used a constant value $F 10.7=130$ sfu corresponding to the mean SA level. This approach was used to isolate the thermo- spheric effect on dynamical processes in the atmosphere. To account for the impact of charged particles on the neutral gas motion at ionospheric heights at different SA levels, MUAM interpreted the geomagnetic torque and ion drag indices, calculated for January in terms of diurnal variations for all latitudes, longitudes, and 23 vertical levels above $100 \mathrm{~km}$ [Shevchuk et al., 2018].

\section{Estimated statistical significance}

To examine the statistical significance of the SA effect on amplitudes of long-period PWs, a paired Student's $t$-test [Rice, 2006; Kobzar, 2006] applied to two sets of amplitude values corresponding to high and low SA was used. At each point of the latitude-height amplitude distribution, a hypothesis was tested that the mean values of the two datasets corresponding to high and low SA differ. Note that the paired Student's $t$-test is valid only if the datasets under study have a normal distribution. To verify this, the goodness of fit $\chi^{2}$, modified to check the normality of distribution [Kobzar, 2006], was applied.

\section{RESULTS}

\section{Amplitudes of long-period PWs}

Characteristics of PWs were calculated from geopotential height fields simulated in MUAM, with each model run covering the same interval from mid-December to the end of February being divided into five 15-day subintervals. For each subinterval, PW amplitudes and phases were computed using a Fourier series expansion in longitude. This allowed us to estimate amplitudes and phases of PWs with different zonal wave numbers and periods exceeding 15 days, referred to as long-period PWs in this paper. The PW parameters were averaged over height-latitude clusters containing nine adjacent points of the model grid, i.e. PW amplitudes were calculated from 720 individual values at each point in latitude and height $(16$ (runs) $\times 5$ (subintervals) $\times 9$ (grid points)). This approach yielded statistically significant differences of mean PW characteristics due to SA effect.

Figure 1 (left panels) depicts latitude-height distributions of amplitudes of geopotential height variations (in geopotential meters [gpm]), caused by long-period PWs with zonal wave numbers $m=1 \div 4$ (PW1-4, $a-d$ respectively) at high SA, which are averaged over 720 values for an altitude range $0-270 \mathrm{~km}$.

The structure of the distribution of the simulated PW amplitudes is generally consistent both with satellite observations (e.g., [Forbes et al., 2002; Mukhtarov et al., 2010]) and with our previous calculations [Koval et al., 2018b]. Some deviations from the observed distributions of PW amplitudes in the high-latitude lower thermosphere may be attributed to the absence of parameterization of the auroral thermosphere heating in MUAM due to dissipation of energy, released during solar wind - Earth's magnetosphere interaction. The long-term PWs are generated in the lower atmosphere and propagate upward. Figure 1 (left) shows that below $90 \mathrm{~km}$ PW amplitudes are higher in middle and high latitudes of the Northern Hemisphere. This is due to the 


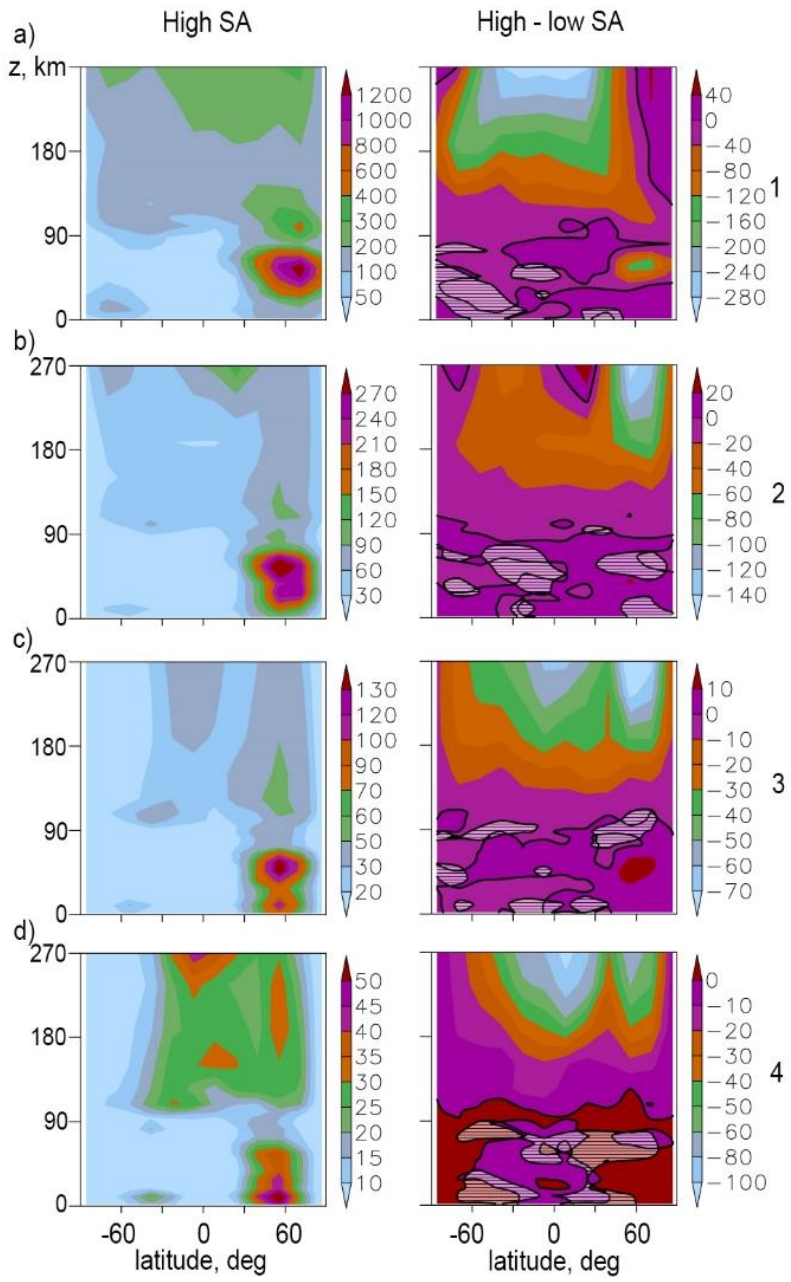

Figure 1. Latitude-height distribution of amplitudes of geopotential height (gpm) variations caused by long-period PWs with zonal wave numbers $1-4$ ( $a-d$ respectively) at high SA (left), and mean differences between PW amplitudes at high and low SA (right) at $0-300 \mathrm{~km}$. Solid contours indicate zero values. Shaded areas are statistically insignificant differences according to the paired Student's $t$-test

fact that in the Northern Hemisphere atmospheric circulation flows in the winter stratomesosphere are eastward, whereas in the Southern Hemisphere the zonal circulation changes its direction with height, thus creating barriers to PW propagation at heights, where the wind changes sign [Charney, Drazin, 1961]. Above 80 $90 \mathrm{~km}$, PWs can penetrate into the Southern Hemisphere, propagating along waveguides [Koval et al., 2018a, b]. With increasing wave number, the waveguides become narrow and PW propagation areas respectively decrease, as PW amplitudes do. The SAdriven changes to dynamical and thermal conditions in the thermosphere cause PW amplitudes to change. The mean differences between PW amplitudes at high and low SA are shown in right panels in Figure 1. The most significant differences between PW amplitudes are observed above $100 \mathrm{~km}$ : SA increase generally leads to their decrease to 50-60\%. The areas with statistically significant data at the $5 \%$ significance level are indicated by hatching. As we can see, these areas appear at heights below $100 \mathrm{~km}$. The statistical significance calculation is described in the next section.
Since this paper examines changes in characteristics of long-period PWs in the middle atmosphere, caused by SA changes at altitudes above $100 \mathrm{~km}$, and the high PW amplitudes in the middle atmosphere take place in the Northern Hemisphere, in the following we will analyze PW characteristics in the Northern Hemisphere.

\section{Estimated statistical significance}

The statistical significance of the differences between simulated PW amplitudes at different SA levels was calculated using the Student's $t$-test [Rice, 2006; Kobzar, 2006]. Its correct application is, however, possible only if the datasets considered have a normal distribution. To check the normality of the amplitude sets, the modified goodness of fit $\chi^{2}$ was applied to each point [Dahiya, Gurland, 1973; Kobzar, 2006].

By way of example, let us check the normality of the PW amplitude set with $m=1$ at one point of the latitudeheight distribution $\left(\varphi=62.5^{\circ} \mathrm{N}, h=8.4 \mathrm{~km}\right)$ during high SA. We have a set of 720 amplitudes: 16 (MUAM runs) $\times 5$ (time intervals from the mid-December to the end of February) $\times 9$ (adjacent points of the MUAM grid).

The mean of the dataset $\bar{x}$ and standard deviation $s$ are calculated from the formulas [Kobzar, 2006]:

$$
\begin{aligned}
& \bar{x}=\frac{1}{n} \sum_{i=1}^{n} x_{i}, \\
& s=\sqrt{d}=\sqrt{\frac{1}{(n-1)} \sum_{i=1}^{n}\left(x_{i}-\bar{x}\right)^{2}},
\end{aligned}
$$

where $n=720$ is the amount of the dataset under study; $d$ is the distribution variance; $x_{i}$ is the PW amplitude at each point. In our case, $\bar{x}=171.04 \mathrm{gpm}, s=74.63$ gpm, $d=5570 \mathrm{gpm}^{2}$.

The dataset is divided into $k=10$ equiprobable intervals, limits of the intervals are defined as

$$
\bar{x}+c_{i} s(i=0, \ldots, 10),
$$

and the coefficients $c_{i}$, symmetric about zero, are taken from Table [Dahiya, Gurland, 1973] for $k=10$.

Figure 2 presents histograms of all 720 PW amplitudes of the set considered: for regular intervals (left) and equiprobable intervals (right) calculated from Formula (2). In the left panel of Figure 2 is a well-defined bell-like shape of the distribution corresponding to the normal distribution, centered at $\bar{x}$. The goodness of fit $\chi^{2}$ is calculated from the formula [Kobzar, 2006]

$$
\chi^{2}=\frac{k}{n} \sum_{i=1}^{k} m_{i}^{2}-n,
$$

where $m_{i}$ is the number of members of the dataset, which falls into the $i$ th equiprobable interval. If $\chi^{2}<d_{\text {crit }}$ of the critical value of the test statistics at the given significance level, the hypothesis of normality of the distribution of the dataset considered is accepted. Table of critical values $d_{\text {crit }}$ is given in [Kobzar, 2006]. Calculate $\chi^{2}$ from Formula (3), obtain $\chi^{2}=5.25$.

According to the Table of critical values $d_{\text {crit }}$ [Kobzar, 2006], at the significance level $\alpha=5 \% d_{\text {crit }}=14.438$. Since $\chi^{2}<d_{\text {crit }}$, the distribution of amplitudes of longperiod PWs can be considered as normal. 

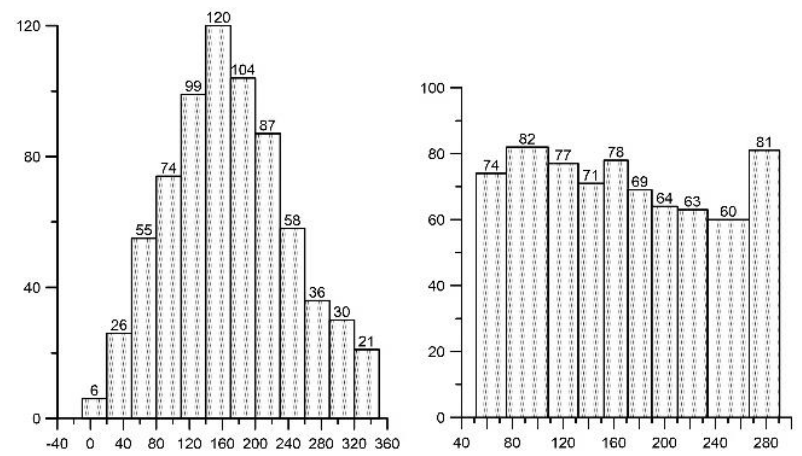

Figure 2. Histograms of $720 \mathrm{PW}$ amplitudes at one point of latitude-height distribution $\left(\varphi=62.5^{\circ} \mathrm{N}, h=8.4 \mathrm{~km}\right)$ constructed at regular intervals (left) and equiprobable intervals (right)

The similar calculation for the amplitudes at low SA, which has shown that the distribution is normal and the calculated variance is similar, $d=5551$, allows us to compute the statistical significance of the difference between mean PW amplitudes, using the Student's $t$-test.

Due to the fact that we examine pairs of PW amplitude sets taken at the same point at the same time under identical initial and background conditions, in this paper we use the paired Student's $t$-test for dependent sets of raw data. The paired Student's $t$-test for dependent samples is calculated from the formula [Rice, 2006]

$$
t=\frac{\bar{X}_{\mathrm{d}}}{s_{\mathrm{d}} / \sqrt{n}},
$$

where $\bar{X}_{\mathrm{d}}$ is the mean differences between corresponding pairs of amplitudes for high and low SA, $s_{\mathrm{d}}$ is the standard deviation of these differences, $n$ is the number of pairs of values. Since the differences at nine adjacent points of the MUAM grid can be correlated, the number of degrees of freedom $f=79$ (16 (MUAM runs) $\times 5$ (intervals) -1 ).

The mean differences $\bar{X}_{\mathrm{d}}$ and the standard deviation $s_{\mathrm{d}}$ are calculated from Formulas (1) applied to the respective amplitude differences at each point. In our case, $n=720 ; \bar{X}_{\mathrm{d}}=9.01 ; s_{\mathrm{d}}=85.11$. Substituting these values into Formula (4), we get $t=2.84$. Next, at the given significance level $\alpha=5 \%$ we determine the null hypothesis $\mathrm{H}_{0}$ that the differences between mean $\mathrm{PW}$ amplitudes $\bar{X}_{\mathrm{d}}=0$, and the alternative $\mathrm{H}_{1}, \bar{X}_{\mathrm{d}} \neq 0$.

The calculated Student's $t$-test is compared with the critical value of the $t$-test for the significance level $\alpha=5$ $\%$ [Doerffel, 1994]. Given $f>60, t_{\text {crit }}=1.96$. Since $t>$ $t_{\text {crit }}$, we can say that at the $5 \%$ significance level the hypothesis $\mathrm{H}_{0}$ is rejected and the hypothesis $\mathrm{H}_{1}$ is accepted, i.e. at the latitude-height distribution point of interest there is a statistically significant non-zero difference between mean amplitudes with a probability of $95 \%$.

Similar calculations have been made for all points of the latitude-height distribution of differences between amplitudes of long-period PWs due to SA effects.

PW amplitudes in the middle atmosphere of the Northern Hemisphere

Figure 3 displays the amplitudes of geopotential height variations calculated in MUAM, which were caused by long-period PWs in the Northern Hemisphere at high SA (left) at 0-100 km, variations in PW amplitudes due to SA-dependent changes in the thermosphere (center), as well as the $t$-test calculated at each point (right).

Figure 3, $a-d$ shows PW1-4. The black contour in right and central panels denotes the critical statistical significance level $t_{\text {crit }}=1.96$. In central panels of Figure 3 , statistically insignificant differences are hatched.

For a more detailed analysis of the model calculations of PW amplitudes, vectors of the Eliassen-Palm flux (EP flux) are calculated $F_{\mathrm{m}}=\left(F_{\mathrm{m}}^{(\varphi}, F_{\mathrm{m}}^{(z)}\right)$. For quasigeostrophic conditions and log-isobaric vertical coordinate the meridional and vertical components of the EP flux (assigned to density) are computed from formulas [Andrews et al., 1987]

$$
\begin{aligned}
& F_{\mathrm{m}}^{(\varphi)}=-a \cos \varphi\left(\overline{u^{\prime} v^{\prime}}\right), \\
& F_{\mathrm{m}}^{(z)}=a f \cos \varphi\left(\overline{v^{\prime} \theta^{\prime}}\right) / \bar{\theta}_{z},
\end{aligned}
$$

where hatches denote disturbances caused by the PW modes considered, $u$ and $v$ are the zonal and meridional winds; $z$ is the vertical coordinate; $\theta$ is the potential temperature; $\varphi$ is the latitude; $a$ is the Earth radius; $f$ is the Coriolis parameter. According to Formula (5), the upward EP flux corresponds to the northward PW heat flux $\overline{v^{\prime} \theta}$, and the southward EP flux matches the northward PW momentum flux $\overline{u^{\prime} v^{\prime}}$. Divergence of the EP flux determines the acceleration of the zonal mean flow generated by PW. Figure 4 shows EP-flux vectors and their increments due to SA changes corresponding to PW1-4, depicted in Figure 3.

As shown above, the maximum PW amplitudes occur at middle latitudes of the Northern Hemisphere. In this case, the maximum PW amplitudes (Figure 3, $a-d$, left panels) are matched by the southward and upward maximum EP fluxes (Figure 4, left panels). Changes in PW amplitudes below $100 \mathrm{~km}$ due to SA thermospheric changes may run to $10-15 \%$ (Figure 3 , middle panels). A significant difference of PW1 (Figure 3,a) from the other PW modes is a decrease in the the amplitude in middle and high latitudes and at altitudes above $40 \mathrm{~km}$ during high SA. This decrease occurs with an increase in PW amplitude at high latitudes in the thermosphere (Figure 1, $a$, right panel).

Such behavior of PW1 can be explained by the fact that at high SA most of the PW1 energy is transferred from the middle atmosphere to the thermosphere, unlike PW2-4 characterized by reflection gain at heights of the lower thermosphere at high SA as compared to low SA. This leads to an increase in amplitudes of these modes in the middle atmosphere and their decrease in the thermosphere. A similar effect was also observed in the previous studies (e.g., [Lu et al., 2017]). In addition to the effect of reflection, PW amplitudes are affected by EP fluxes. The northward and downward vectors of EPflux increments correspond to deterioration of PW propagation conditions at 40-70 km (Figure 3, a, d, middle panels), which in turn leads to a decrease in PW1 and PW4 amplitudes, whereas upward and southward vectors of EP-flux increments correspond to 

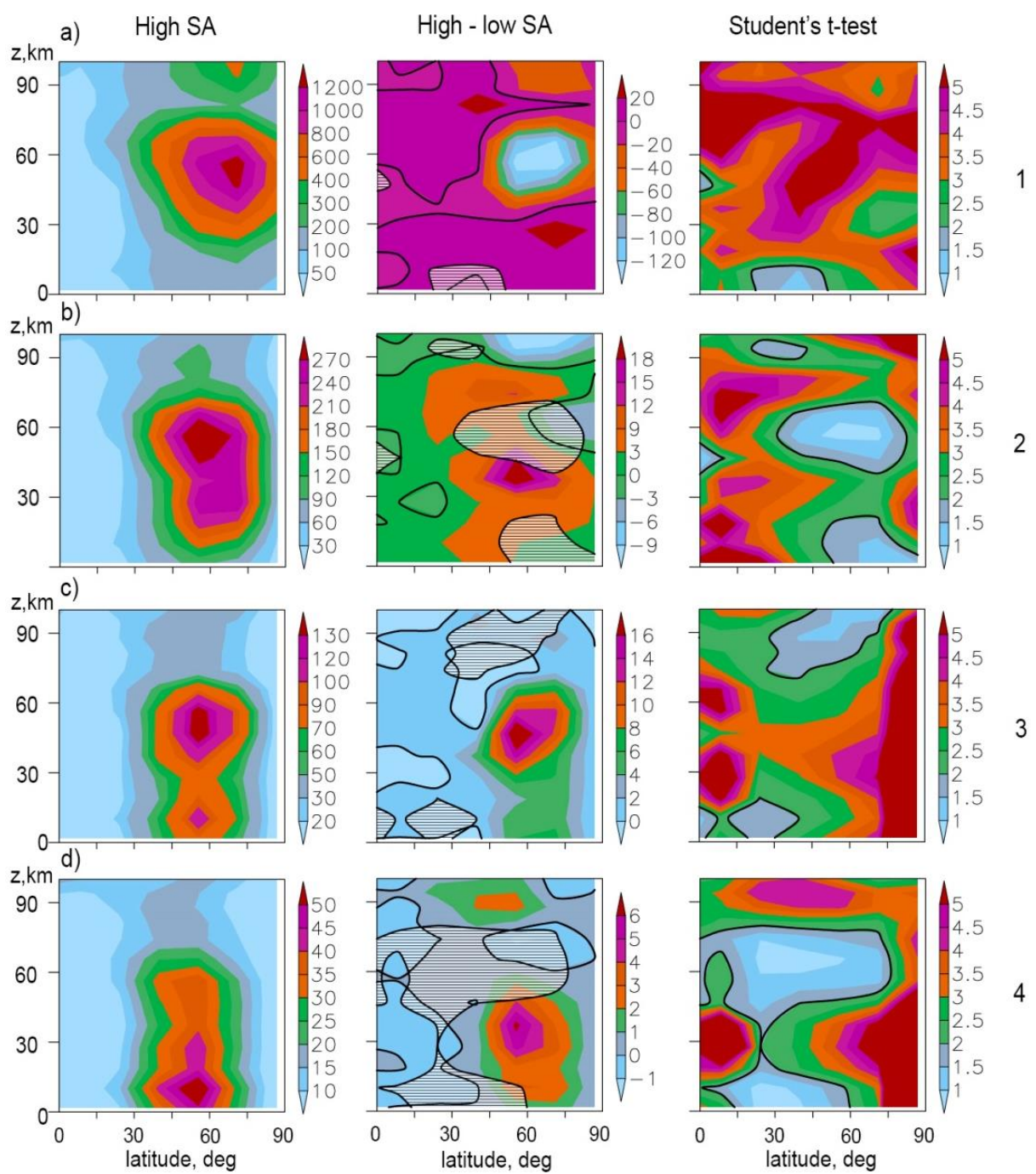

Figure 3. Amplitudes of geopotential height (gmp) variations driven by long-period PWs with zonal wave numbers 1-4 ( $a-d$ respectively) at high SA (left), differences between PW amplitudes at high and low SA (center), and values of the Student's $t$-test calculated from 720 differences of value pairs (right) for the Northern Hemisphere at $0-100 \mathrm{~km}$. Solid contours indicate zero values. Solid contours in right panels correspond to the hatched areas in the middle ones and represent statistically insignificant differences

improvement of $\mathrm{PW}$ propagation conditions, which leads to an increase in PW amplitudes. Below $40 \mathrm{~km}$, all the above PW modes feature a gain to $5-10 \%$ in middle and high latitudes of the Northern Hemisphere at high SA, which is caused particularly by an enhancement of upward EP fluxes in these regions.

Calculation of the statistical significance for nonzero differences in PW amplitudes (Figure 3, middle panels) at a significance level of $5 \%$ has shown that statistically significant results have been obtained for most areas of the distributions considered.

The analysis of PW phases has revealed a phase shift up to $150^{\circ}$ for PW1 at $80-100 \mathrm{~km}$, which supports the hypothesis that PWs propagate into the lower ionosphere not directly but either through excitation of secondary waves due to tidal dissipation [Laštovicka, 2006] or through diffusion of mesospheric atomic oxygen in the ionosphere [Pancheva, Lysenko, 1988].

\section{CONCLUSION}

The model of the middle and upper atmosphere MUAM has been used to carry out the numerical simulation of the general atmospheric circulation at altitudes to $300 \mathrm{~km}$. Amplitudes of geopotential height variations caused by long-period PWs have been calculated. On the basis of two ensembles of 16 model runs, we have obtained statistically significant results of calculations of SAdependent variations in PW amplitudes. To study the thermospheric effect of SA in MUAM above $100 \mathrm{~km}$, different values of the solar radio flux with a wavelength of $10.7 \mathrm{~cm}$ were set and geomagnetic torque and ion drag indices corresponding to different SA levels were integrated.

The numerical simulations have shown that the consideration of SA increase for altitudes above $100 \mathrm{~km}$ leads to a statistically significant decrease in amplitudes of longperiod PWs to $60 \%$ in the thermosphere and to $10-15 \%$ in the middle atmosphere. A significant contribution to 


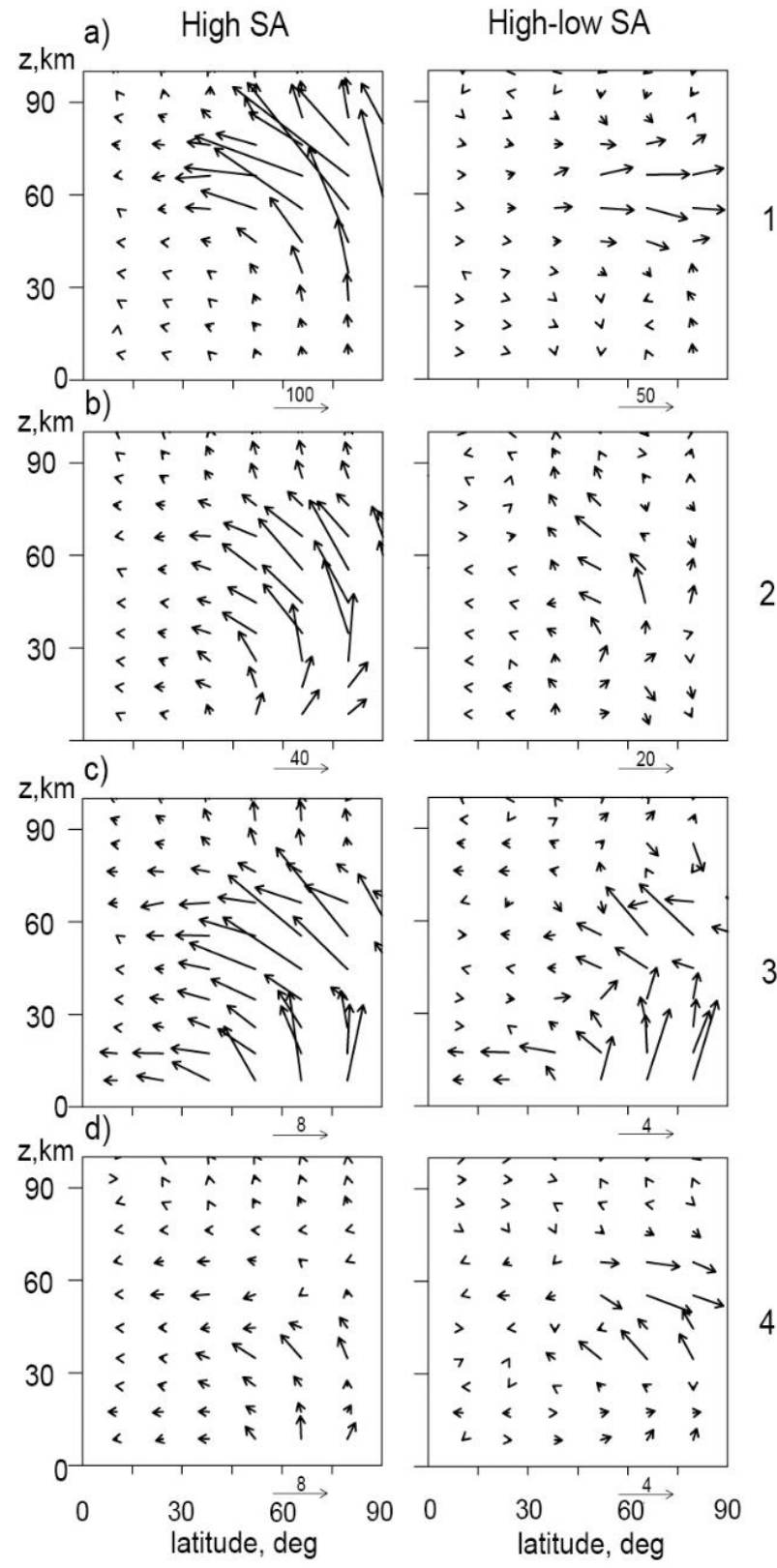

Figure 4. Vectors of the Eliassen-Palm flux (in $\mathrm{m}^{3} / \mathrm{s}^{2}$ ) for high SA (left) and flux increments due to SA change (right) corresponding to PW1-4 in Figure 3. For clarity, the vertical component is multiplied by 200

PW2-4 propagation at high SA is made by reflection of PW energy at MLT heights, leading to an increase in the amplitudes of these waves in the middle atmosphere of the Northern Hemisphere and to a decrease in the amplitudes in the thermosphere. The PW1 energy is transferred from the middle atmosphere to the thermosphere, leading to the attenuation of PW1 in middle and high latitudes of the Northern Hemisphere in the middle atmosphere and to their amplification in the thermosphere.

To gain a better understanding of PW propagation conditions, EP fluxes corresponding to PWs have been calculated. The amplification of upward and southward EP fluxes is followed by an increase in PW amplitudes in respective regions, and the attenuation of these fluxes is accompanied by a decrease in PW amplitudes, particularly notable for PW1.
The results show for the first time with a high degree of statistical confidence that changes in dynamic and thermal regimes of the thermosphere during SA changes significantly affect characteristics of long-period PWs in the middle atmosphere. PW propagation in the middle atmosphere is materially affected by wave reflection at MLT heights.

This work was supported by the Russian Science Foundation (grant No. 18-77-00022).

\section{REFERENCES}

Andrews D.G., Holton J.R., Leovy C.B. Middle Atmosphere Dynamics. New York, Acad. Press, 1987. 489 p.

Arnold N.F., Robinson T.R. Solar cycle changes to planetary wave propagation and their influence on the middle atmosphere circulation. Ann. Geophys. 1998, vol. 16, iss. 1, pp. 69-76. DOI: 10.1007/s00585-997-0069-3.

Bruevich E.A., Yakunina G.V. The cyclic activity of the sun from observations of the activity indices at different time scales. Moscow University Physics Bulletin. 2015, vol. 70, iss. 4, pp. 282-290.

Chang L.C., Yue L., Wang W., Wu Q., Meier R.R. Quasi two day wave-related variability in the background dynamics and composition of the mesosphere/thermosphere and the ionosphere. J. Geophys. Res.: Space Phys. 2014, vol. 119, iss. 6, pp. 4786-4804. DOI: 10.1002/2014JA019936.

Charney J.G., Drazin P.G. Propagation of planetary-scale disturbances from the lower into the upper atmosphere. $J$. Geophys. Res. 1961, vol. 66, no. 1, pp. 83-109.

Dahiya R.C., Gurland J. How many classes in the Pearson Chi-square test? Journal of the American Statistical Association. 1973, vol. 68, no. 343, pp. 707-712. DOI: 10.2307/2284803.

Doerffel K. Statistika v analiticheskoi khimii [Statistics in analytical chemistry: translation from German]. Moscow, Mir, 1994. 268 p. (in Russian). (German edition: Doerffel K. Statistik in der analytischen Chemie. Leipzig, VEB Deutscher Verlag für Grundstoffindustrie, 1982.)

Forbes J. M., Zhang X., Ward W., Talaat E.R. Climatological features of mesosphere and lower thermosphere stationary planetary waves within \pm 40 latitude. J. Geophys. Res. 2002, vol. 107, iss. D17, 4322. DOI: 10.1029/2001JD001232.

Fröhlich K., Pogoreltsev A., Jacobi Ch. Numerical simulation of tides, Rossby and Kelvin waves with the COMMALIM model. Adv. Space Res. 2003, vol. 32, iss. 5, pp. 863-868. DOI: $10.1016 / \mathrm{S} 0273-1177(03) 00416-2$.

Gavrilov N.M. Parameterization of momentum and energy depositions from gravity waves generated by tropospheric hydrodynamic sources. Ann. Geophys. 1997, vol. 15, iss. 12, pp. 1570-1580. DOI: 10.1007/s00585-997-1570-4.

Gavrilov N.M., Koval A.V. Parameterization of mesoscale stationary orographic wave forcing for use in numerical models of atmospheric dynamics. Izvestiya. Atmos. Ocean. Phys. 2013, vol. 49, iss. 3, pp. 244-251. DOI: 10.1134/S0001433813030067.

Gavrilov N.M., Pogoreltsev A.I., Jacobi Ch. Numerical modeling of the effect of latitude-inhomogeneous gravity waves on the circulation of the middle atmosphere. Izvestiya. Atmos. Ocean. Phys. 2005, vol. 41, iss. 1, pp. 9-18.

Geller M. A., Alpert J.C. Planetary wave coupling between the troposphere and the middle atmosphere as a possible Sun-weather mechanism. J. Atmos. Sci. 1980, vol. 37, pp. 1197-1215.

Hathaway D.H. The Solar Cycle. Living Rev. Solar Phys. 2010, vol. 12, 4. DOI: 10.1007/lrsp-2015-4.

Holton J.R. The Dynamic Meteorology of the Stratosphere and Mesosphere. 1975, 218 p. (Meteorol. Monographs, vol. 15, no. 37). 
Jacobi Ch., Hoffmann P., Kurschner D. Trends in MLT region winds and planetary waves, Collm $\left(52^{\circ} \mathrm{N}, 15^{\circ} \mathrm{E}\right)$. Ann. Geophys. 2008, vol. 26, iss. 5, pp. 1221-1232. DOI: 10.5194/angeo26-1221-2008.

Kobayashi S., Ota Y., Harada H., Ebita A., Moriya M., Onoda H., Onogi K., et al. The JRA-55 Reanalysis: general specifications and basic characteristics. J. Meteorol. Soc. Japan. 2015, vol. 93, iss. 1, pp. 5-48. DOI: 10.2151/jmsj.2015-001.

Kobzar A.I. Prikladnaya matematicheskaya statistika [Applied mathematical statistics]. Moscow, Fizmatlit Publ., 2006, 816 p. (In Russian).

Koval A.V., Gavrilov N.M, Pogoreltsev A.I., Shevchuk N.O. Influence of solar activity on penetration of traveling planetaryscale waves from the troposphere into the thermosphere. $J$. Geophys. Res.: Space Phys. 2018a, vol. 123, iss. 8, pp. 6888-6903. DOI: 10.1029/2018JA025680.

Koval A.V., Gavrilov N.M., Pogoreltsev A.I., Shevchuk N.O. Propagation of stationary planetary waves to the thermosphere at different levels of solar activity. J. Atmos. Solar-Terr. Phys. 2018b, vol. 173, pp. 140-149. DOI: 10.1016/j.jastp.2018.03.012.

Krivolutsky A.A., Cherepanova L.A., Dement'eva A.V., Repnev A.I., Klyuchnikova A.V. Global circulation of the Earth's atmosphere at altitudes from 0 to $135 \mathrm{~km}$ simulated with the ARM model. Consideration of the solar activity contribution. Geomagnetism and Aeronomy. 2015, vol. 55, iss. 6 , pp. 780-800. DOI: 10.1134/S0016793215060067.

Laštovicka J. Forcing of the ionosphere by waves from below. J. Atmos. Sol.-Terr. Phys. 2006, vol. 68, iss. 3, pp. 479-497.

Liu H.L., Talaat E.R., Roble R.G., Lieberman R.S., Riggin D.M., Yee J.H. The 6.5-day wave and its seasonal variability in the middle and upper atmosphere. J. Geophys. Res.: Atmos. 2004, vol. 109, iss. D21, D21112. DOI: 10.1029/2004jd004795.

Lu H., Scaife A.A., Marshall G.J., Turner J., Gray L.J. Downward wave reflection as a mechanism for the stratospheretroposphere response to the 11-year solar cycle. J. Clim. 2017, vol. 30, no. 7, pp. 2395-2414. DOI: 10.1175/JCLI-D-16-0400.1.

Mukhtarov P., Pancheva D., Andonov B. Climatology of the stationary planetary waves seen in the SABER/TIMED temperatures (2002-2007). J. Geophys. Res. 2010, vol. 115, A06315. DOI: 10.1029/2009JA015156.

Pancheva D., Lysenko I. Quasi-two-day fluctuations ob- served in the summer $\mathrm{F}$ region electron maximum. Bulg. Geophys. J. 1988, vol. 14, no. 2, pp. 41-51.

Pogoreltsev A.I. Generation of normal atmospheric modes by stratospheric vacillations. Izvestiya. Atmos. Ocean. Phys. 2007, vol. 43, no. 4, pp. 423-435. DOI: 10.1134/S0001433807040044.

Pogoreltsev A.I., Vlasov A.A., Fröhlich K., Jacobi Ch. Planetary waves in coupling the lower and upper atmosphere. J. Atmos. Solar-Terr. Phys. 2007, vol. 69, iss. 17-18, pp. 2083-2101. DOI: 10.1016/j.jastp.2007.05.014.

Rice J.A. Mathematical Statistics and Data Analysis. 3rd edition. Pacific Grove. Duxbury Press, 2006, 603 p.

Richards P.G., Fennelly J.A., Torr D.G. EUVAC: a solar EUV flux model for aeronomic calculations. J. Geophys. Res. 1994, vol. 99, iss. A5, pp. 8981-8992. DOI: 10.1029/94JA00518.

Shevchuk N. O., Ortikov M. Yu., Pogoreltsev, A. I. Modeling of atmospheric tides with account of diurnal variations of ionospheric conductivity. Russian Journal of Physical Chemistry B. 2018, vol. 12, no. 3, pp. 576-589. DOI: $10.1134 /$ S199079311803017X.

Tapping K.F. Recent solar radio astronomy at centimeter wavelength: The temporal variability of the $10.7-\mathrm{cm}$ flux. $J$. Geophys. Res.: Atmos. 1987, vol. 92, iss. D1, pp. 829-838. DOI: 10.1029/JD092iD01p00829.

Vitinsky Yu.I., Kopetsky M., Kuklin G.V. Statistika pyatnoobrazovatel'noi deyatel'nosti Solntsa [Statistics of sunspot-forming activity of the Sun]. Moscow, Nauka Publ., 1986, 296 p. (In Russian).

Wang J.C., Chang L.C., Yue J., Wang W., Siskind D.E. The quasi 2 day wave response in TIME-GCM nudged with NOGAPS-ALPHA. J. Geophys. Res.: Space Phys. 2017, vol. 122, iss. 5, pp. 5709-5732. DOI: 10.1002/2016JA023745.

URL: http://sidc.be/silso/datafiles (accessed November 18, 2017).

How to cite this article

Koval A.V. Statistically significant estimates of influence of solar activity on planetary waves in the middle atmosphere of the Northern Hemisphere as derived from MUAM model data. Solar-Terrestrial Physics. 2019. Vol. 5. Iss. 4. P. 53-59. DOI: 10.12737/stp-54201907. 